Article

\title{
Prediction of Health Care Costs by Dental Health Care Costs and Periodontal Status
}

\author{
Yoshiaki Nomura ${ }^{1, *(D)}$, Tetsuro Sato ${ }^{2}$, Yoshinori Kamoshida ${ }^{2}$, Syunsuke Suzuki ${ }^{2}$, \\ Ayako Okada ${ }^{3}$, Ryoko Otsuka ${ }^{1}$, Erika Kakuta ${ }^{4}$ and Nobuhiro Hanada ${ }^{1}$ \\ 1 Department of Translational Research, Tsurumi University School of Dental Medicine, 2-1-3 Tsurumi, \\ Tsurumi-ku, Yokohama 230-8501, Japan; otsuka-ryoko@tsurumi-u.ac.jp (R.O.); \\ hanada-n@tsurumi-u.ac.jp (N.H.) \\ 2 Kanagawa Dental Association, 6-68 Sumiyoshi-cho Cyuuou-ku, Yokohama 231-0016, Japan; \\ kumasan@r6.ucom.ne.jp (T.S.); phdkamo@gray.plala.or.jp (Y.K.); shun-s@wg8.so-net.ne.jp (S.S.) \\ 3 Department of Operative Dentistry, Tsurumi University School of Dental Medicine, 2-1-3 Tsurumi, \\ Tsurumi-ku, Yokohama 230-8501, Japan; okada-a@tsurumi-u.ac.jp \\ 4 Department of Oral Bacteriology, Tsurumi University School of Dental Medicine, 2-1-3 Tsurumi, Tsurumi-ku, \\ Yokohama 230-8501, Japan; kakuta-erika@tsurumi-u.ac.jp \\ * Correspondence: nomura-y@tsurumi-u.ac.jp; Tel.: +81-45-580-8462; Fax: +81-45-573-2473
}

Received: 27 March 2020; Accepted: 25 April 2020; Published: 30 April 2020

\begin{abstract}
Reducing heath care costs is an important issue in Japan. The aim of this study was to analyze the contribution of oral health to health care costs and to predict health care costs by statistical modeling. Data from 46 individuals ( 29 men and 17 women; mean age of $44.6 \pm 1.7$ years) on health care costs, dental health care costs, and the results of the salivary levels of lactate dehydrogenase (LD) over two years were provided by the association. Multilayer perceptron neural networks were applied to predict the health care costs from data from the previous year and included health care costs, dental health care costs, and salivary levels of LD. Nonlinear relationships were observed between medical health care costs, dental health care costs, and periodontal conditions. The health care costs from the previous year were the most important predictor of health care costs. The simulation results showed that health care costs decreased with the increase in dental health care costs from the previous year. Health care costs increased with increasing salivary levels of LD from the previous year. Improvements in periodontal conditions and dental health care may play some roles in reducing health care costs.
\end{abstract}

Keywords: health care cost; saliva; periodontal disease; neural network

\section{Introduction}

High health care costs are a major problem in Japan. National medical expenditure in 2016 was 382 billion USD (42 trillion yen), 300 USD (33,000 yen) per capita, and 7.81\% of Gross Domestic Product [1]. The population of Japan is rapidly aging, which is increasing health care costs. We have a national insurance system covering almost all the treatments for injuries and diseases. The provision of funds for this national insurance system is supported in part by public expense.

Oral health status may contribute to overall health conditions through nutritional status and dental bacteremia. In fact, some reports have shown that subjects with many teeth are expected to live longer than subjects with few or no teeth, mainly through adequate nutritional status [2-4]. Periodontal disease can be a risk factor for various diseases, including noncommunicable diseases [5-7]. Oral bacteria that invade blood vessels can damage body tissues and cause low-grade but persistent inflammation [8]. It has been suggested that Streptococcus mutans, a major cariogenic bacterium, may 
cause atherosclerosis [9-11]. Based on these facts, oral health status may be related to health care costs. The treatment and prevention of dental diseases may lead to a decrease in medical costs.

Since 2000, the Japanese government has planned and implemented government policies to extend healthy longevity through health promotion. This plan is called Healthy Japan 21. The main target of this plan is the prevention of noncommunicable diseases. In this declaration, to prevent noncommunicable diseases, dental health promotion is included in the six main pillars as follows: nutrition and dietary habits, physical activity and exercise, rest, drinking of alcohol, smoking of tobacco, and oral health [12].

In addition, the Ministry of Health, Labor, and Welfare stipulates that the insurer must analyze medical costs and the results of the annual health checkup to make plans to prevent a rise in medical costs. Prediction and simulation must prevent a rise in medical costs. However, there is little information on the correlation between medical health care costs, dental health care costs, and oral health status. The main reason is that it is almost impossible to calculate and analyze health care costs without a national insurance system for the whole nation.

The implementation of dental checkups in annual health checkups is a burden for insurers because of the high costs of dental work. Therefore, to evaluate the condition of periodontal disease, the salivary levels of lactate dehydrogenase (LD) have been adopted as a biomarker [10]. In previous studies, the salivary concentration of LD correlated with the state of periodontal disease, and the value of LD has been shown to reflect inflammation of periodontal tissue [13-15].

The purpose of this study was to analyze the contribution of dental health care costs and oral hygiene to health care costs and to predict health care costs by statistical modeling. Predictive models can lead to the promotion of treatment for dental diseases to prevent systemic diseases, which ultimately leads to preventing an increase in health care costs.

\section{Methods}

\subsection{Study Design}

The study was an observational longitudinal study. All employees of an association were followed up for two years. Annual medical and dental costs were obtained from the insurer. Saliva tests were carried out at the annual health checkup.

\subsection{Participants}

The subjects who participated in this study were individuals who worked for a health insurance association in the Kanagawa Prefecture in Japan. This prefecture is located next to Tokyo, which is the capital of Japan. Informed written consent was obtained from all the subjects.

\subsection{Measuring Salivary Levels of Lactate Dehydrogenase (LD)}

The association conducted a health check every year, including a dental examination in April. The measurement of the saliva concentration of LD was applied instead of a periodontal examination by pocket probing. Saliva samples were taken prior to oral clinical examinations.

According to the manufacturer's instructions, saliva samples were collected at least $2 \mathrm{~h}$ after eating, drinking, or tooth brushing. Participants were instructed to chew on a standard-sized tasteless and odorless gum base for $5 \mathrm{~min}$, during which time stimulated whole saliva was continuously collected. Saliva was kept at $4{ }^{\circ} \mathrm{C}$. Salivary levels of LD were measured using commercially available kits (L type Wako LDH J; Wako Chemical Industry, Osaka, Japan) [13-15].

\subsection{Data Collection on Health Care Costs}

The association provided data on medical expenses, dental expenses, and the results of saliva concentrations of LD over 2 years. Data on health care costs were provided as an annual sum of expenses. 


\subsection{Statistical Method}

Neural network models are very flexible in building predictive models. Both continuous and discrete variables can be used as both predictor and explanatory variables. In addition, these models can predict nonlinear relationships. Previous reports showed that a nonlinear relationship could be detected between oral condition and health care costs [16]. The statistical software creates the optimal number of hidden layers and automatically weights the relationship between explanatory variables and hidden layers, and the relationship between predictors and hidden layers.

Health care costs included medical health care costs and dental health care costs. The multilayer perceptron neural network was applied using health care costs (2017) as the dependent variable. Health care costs (2016), dental care costs (2016) and salivary levels of LD in the saliva (2016) were used as independent variables.

Analyses were carried out using IBM SPSS Statistics V 24.0 and Modeler V 25.0 (IBM SPSS, Tokyo, Japan).

\section{Results}

The study population was 46 individuals ( 29 men and 17 women; mean age pf $44.6 \pm 1.7$ years). A descriptive analysis of the data used in this study is shown in Table 1. The standard deviation values were high, and their distributions were skewed. In the Kolmogorov-Smirnov test, none of these values were normally distributed.

Table 1. Descriptive analysis of the health care costs and salivary levels of lactate dehydrogenase (LD).

\begin{tabular}{cccc}
\hline & \multicolumn{2}{c}{ Mean \pm SD } & Median (25th-75th Percentile) \\
\hline $\mathbf{2 0 1 6}$ & & & \\
Health care costs (USD) & 1035.00 & \pm 1300.27 & $572.45(220.36-1255.09)$ \\
Medical health care costs (USD) & 877.27 & \pm 248.55 & $482.36(126.91-1161.73)$ \\
Dental health care costs (USD) & 157.73 & \pm 2.39 & $84.55(0-279.55)$ \\
Salivary levels of LD (IU/mL) & 4.09 & \pm 2.39 & $3.70(1.73-5.94)$ \\
2017 & & & \\
Health care costs (USD) & 994.00 & \pm 1172.27 & $550.45(122-1577.27)$ \\
Medical health care costs (USD) & 775.55 & \pm 975.55 & $42.65(105.45-1165.73)$ \\
Dental health care costs (USD) & 218.36 & \pm 402.64 & $0(0-455.91)$ \\
\hline
\end{tabular}

Health care costs are the sum of medical health care costs and dental health care costs. One hundred and ten Japanese yen exchanged for 1 USD. LD: Lactate dehydrogenase.

Figure 1 shows a scatter plot of health care costs (2017) against health care costs (2016), dental health care costs (2016), and salivary levels of lactate dehydrogenase (LD) (2016). The coefficient of determination was not sufficient to create a regression model for predicting health care costs. A nonlinear prediction model based on a neural network model was applied. For the prediction of health care costs (2017), we first constructed models with a single independent variable of health care costs from the previous year (2016), dental health care costs from the previous year (2016), or salivary levels of LD from the previous year (2016) (Figure 2A-C).

Health care costs from the previous year were a strong predictor for health care costs, and the inclusion of dental health care costs and salivary levels of LD for health care costs improved the prediction accuracy. Predictive values were calculated by the models shown in Figure 2A-D.

Next, we used all three independent variables to build a health care cost prediction model (Figure 2D). Predictive accuracy was improved by including dental health care costs and salivary levels of LD in addition to health care costs from the previous year (Figure 3, solid circle and empty circle). 


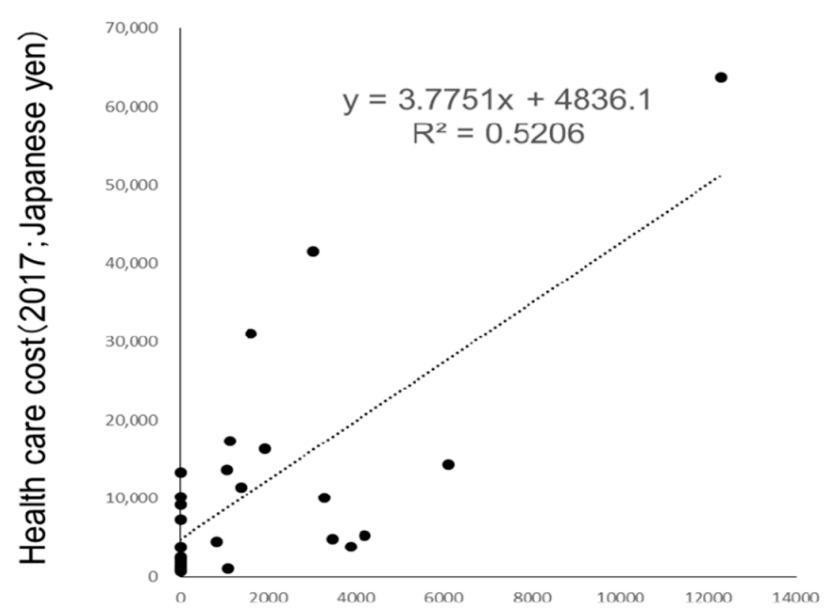

Health care cost (2016; Japanese yen)

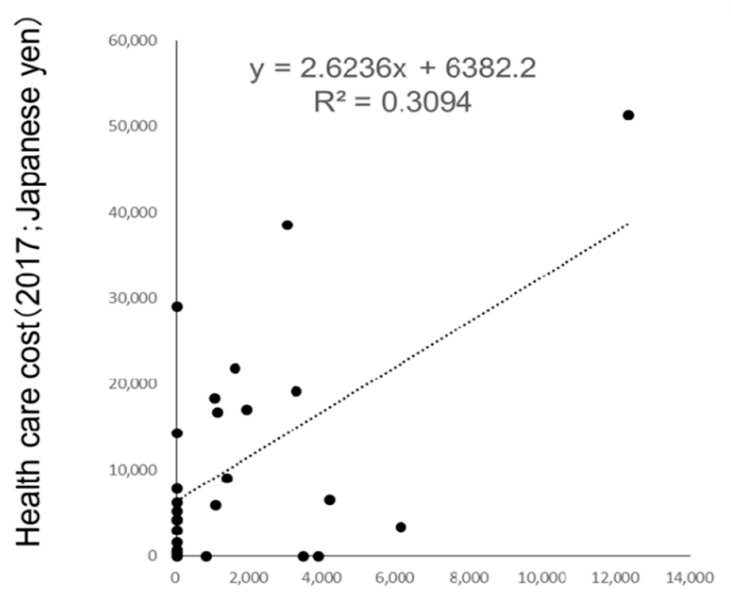

Dental care cost(2016; Japanese yen)

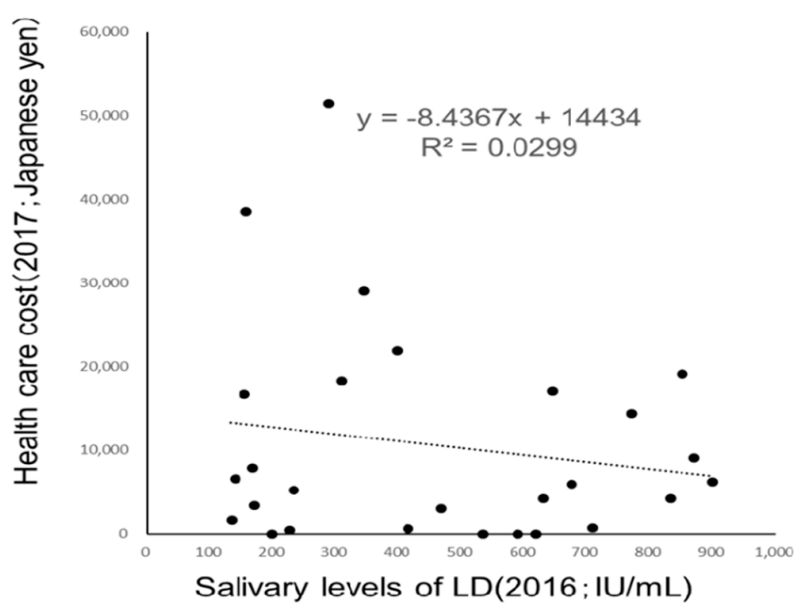

Figure 1. Scatter plot and linear regression line of health care costs against total health care costs, dental health care costs, and salivary levels of lactate dehydrogenase (LD). The coefficient of determination $\left(\mathrm{R}^{2}\right)$ was not high. Health care costs were not easy to predict by linear regression analyses. 

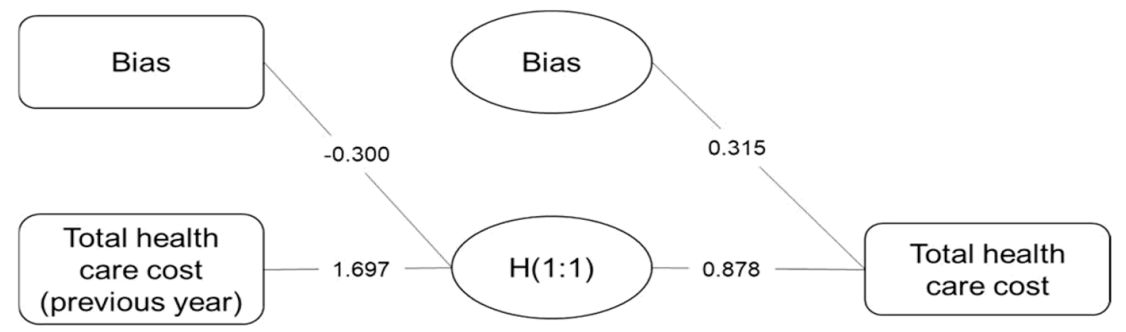

(A)
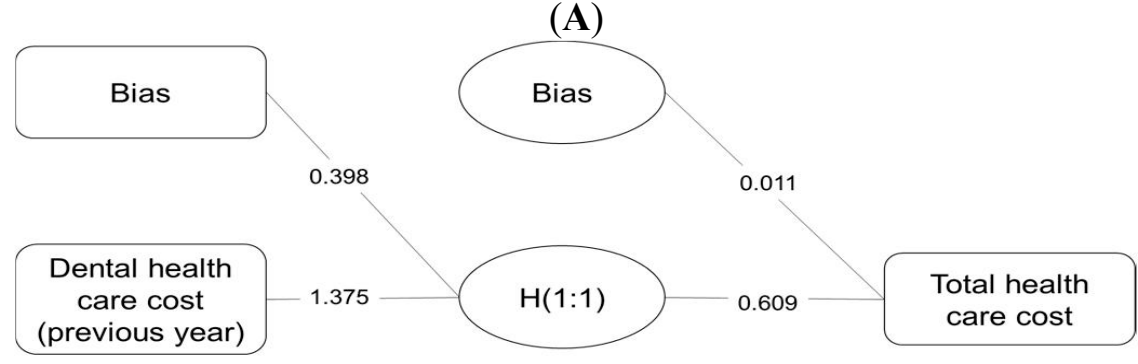

(B)

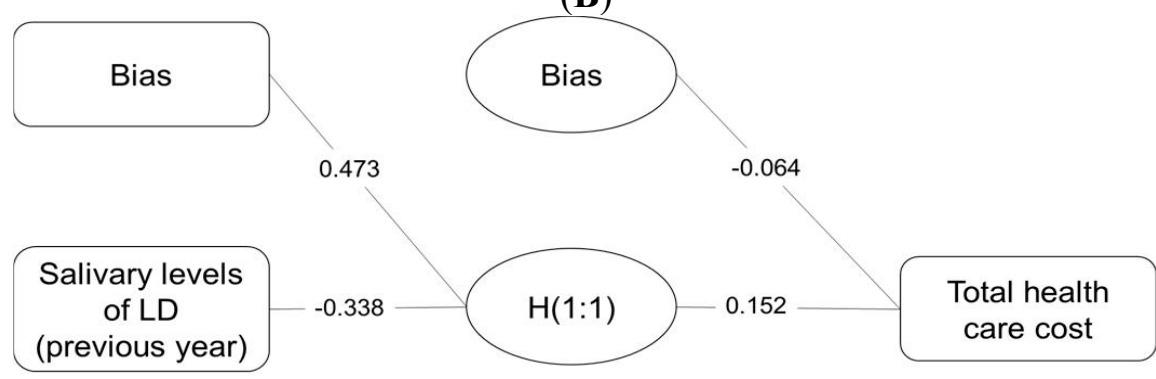

(C)

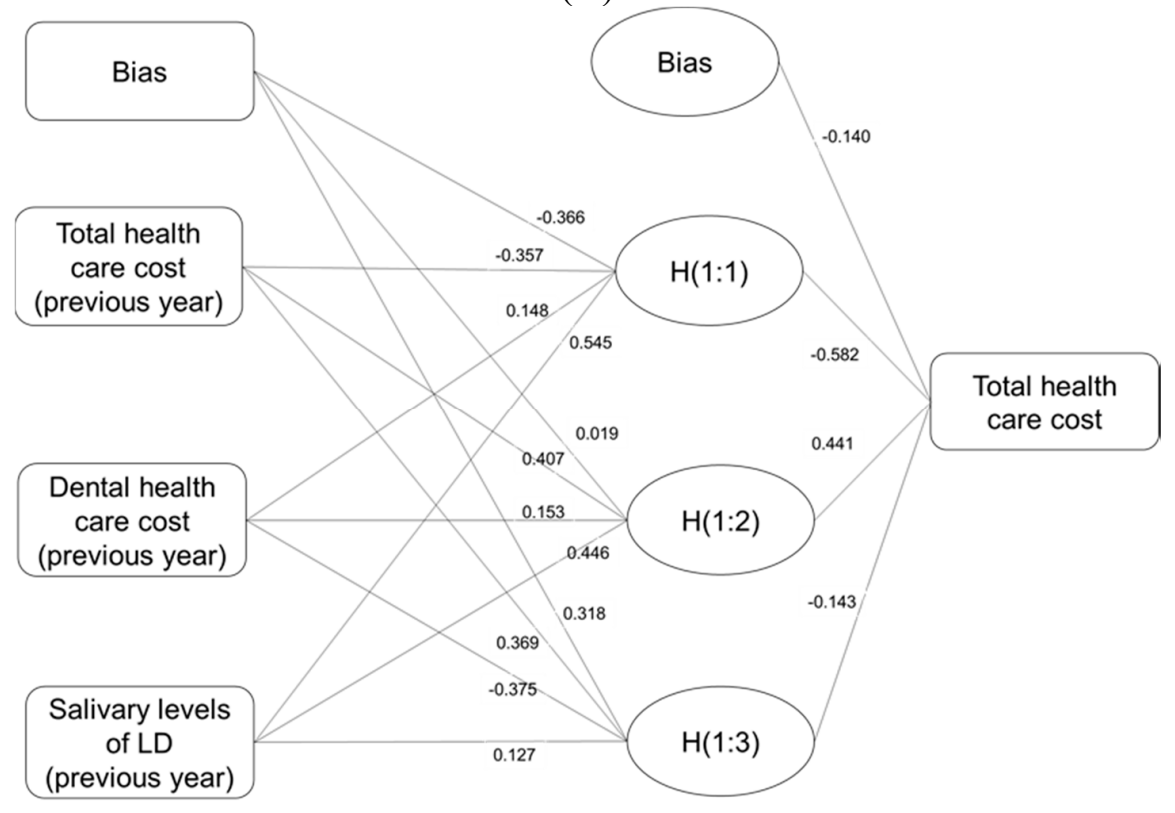

(D)

Figure 2. Models by a single independent variable. (A) health care costs from the previous year; (B) dental health care costs from the previous year; (C) salivary levels of LD from the previous year (2016); (D) Models by multiple these three independent variable. 
The predicted values are shown in the scatter plot of the prediction values against the observed values (Figure 3). Among the three independent variables, health care costs from the previous year were the most important predictor of health care costs.

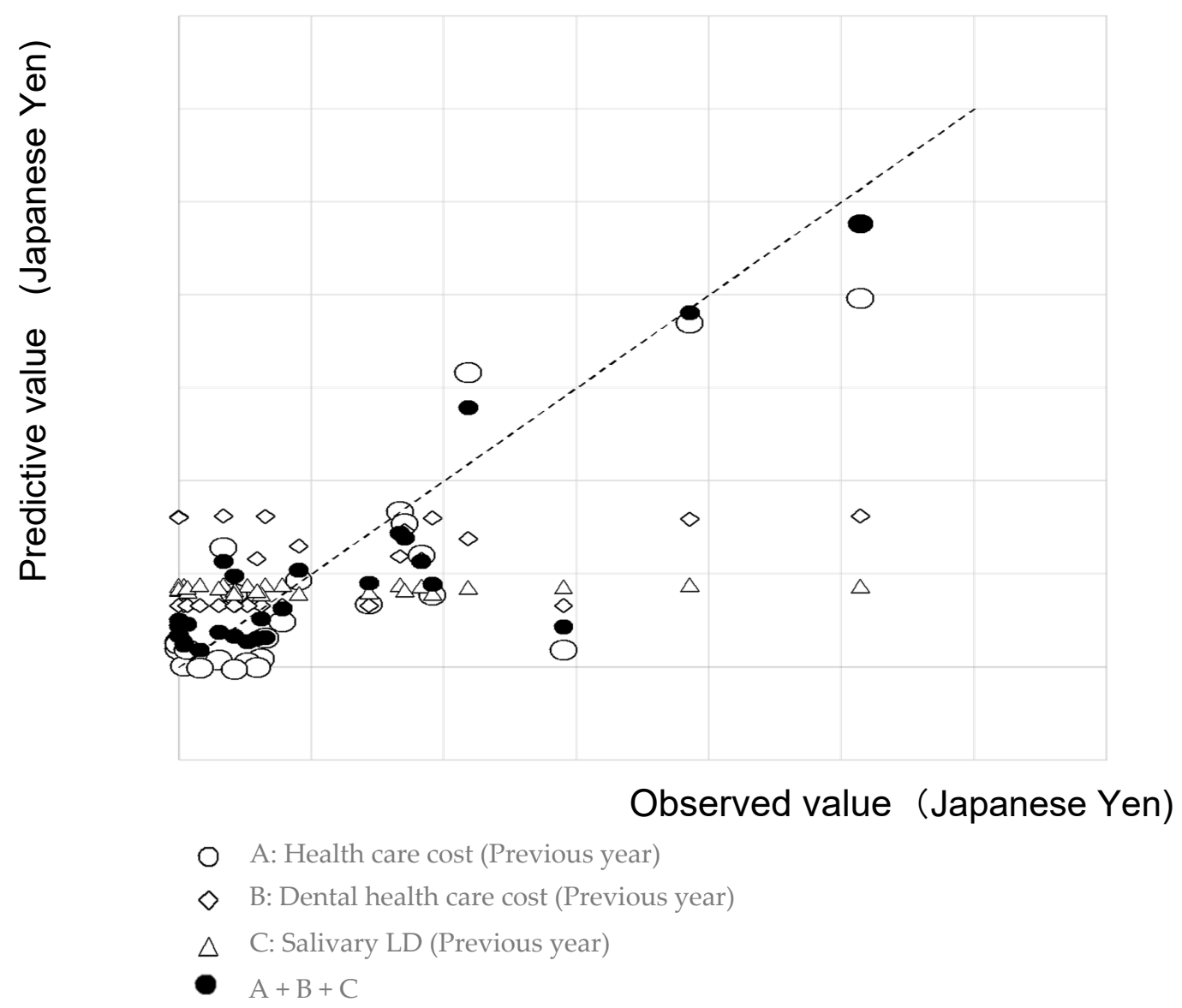

Figure 3. Plot of the predictive values of health care costs against the observed values.

Then, by using the model in Figure 2D, we simulated the health care costs by health care costs, dental health care costs, and salivary levels of LD from the previous year. Since the vector space was four-dimensional, the results are displayed conditionally with the fixed healthcare cost data at 0 , $100,000,200,000,500,000$, and 1 million Japanese yen. The results of the response surfaces are illustrated in Figure 4.

In the case of healthcare costs of 0 yen, if the LD levels in saliva are 0 , then the medical expenses will be 0 yen, and the health care costs the following year will be predicted near the flat surface. As LD levels or dental health care costs increased, health care costs increased, but the gradient was moderate.

Very large pits can be observed around the diagonal by the LD levels and dental health care costs. Pits can be observed in all response surfaces shown in Figure 4. With health care costs of 100,000 yen, 200,000 yen, and 500,000 yen from the previous year, the gradient of the response surface from the start point to the pit became sharp as the health care costs from the previous year increased. For predicted health care costs over the diagonal line, health care costs decreased with increasing LD levels or dental health care costs. For the health care costs from the previous year of 1 million Japanese yen, a sharp decrease in health care costs was observed with the increase in dental health care costs.

Predictive values were calculated by the model shown in Figure 2D. For the prediction, health care costs from the previous year were fixed, and response surfaces were illustrated against dental health care costs and salivary levels of LD from the previous year. Additionally, prediction model for 
the medical care cost (Subtracted dental care cost from total health care cost) was constructed by the same strategy. Results of the model and simulation were shown in Figure 5.

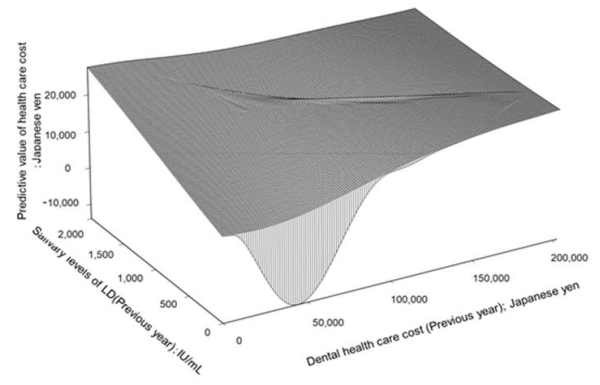

Health care costs from the previous year $=0$ (Japanese Yen)

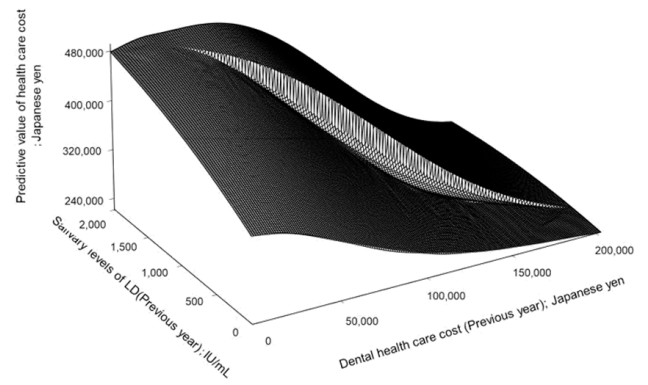

Health care costs from the previous year $=500,000$ (Japanese Yen)
(A)

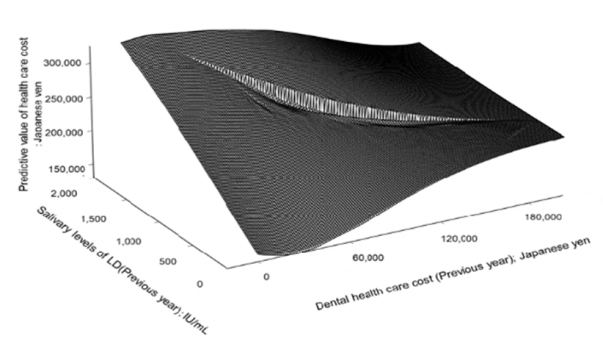

(B)
(D)

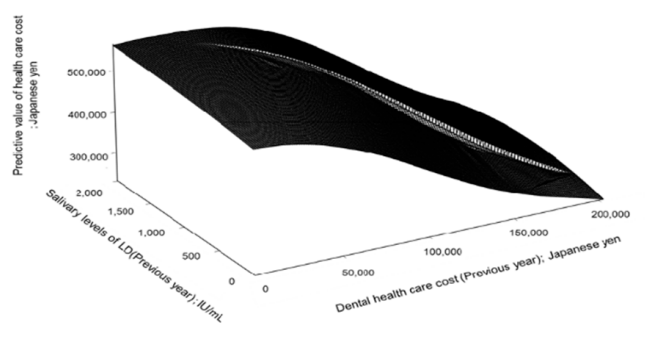

$(\mathbf{E})$

Health care costs from the previous year $=100,000$ Health care costs from the previous year $=1,000,000$ (Japanese Yen) (Japanese Yen)

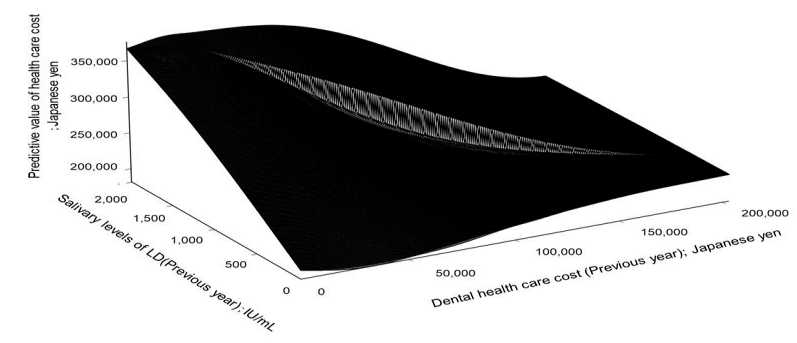

(C)

Health care costs from the previous year $=200,000$ (Japanese Yen)

Figure 4. Results of the simulations of predictive values of health care costs by health care costs, dental health care costs, and salivary levels of LD. 


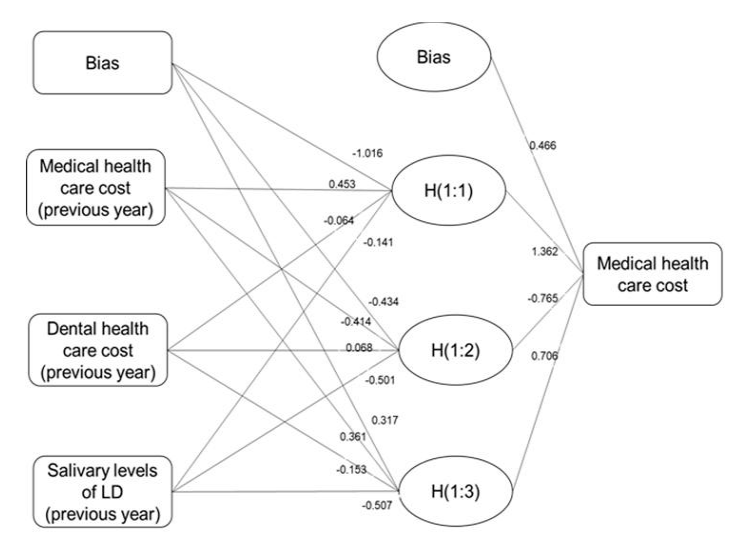

(A)

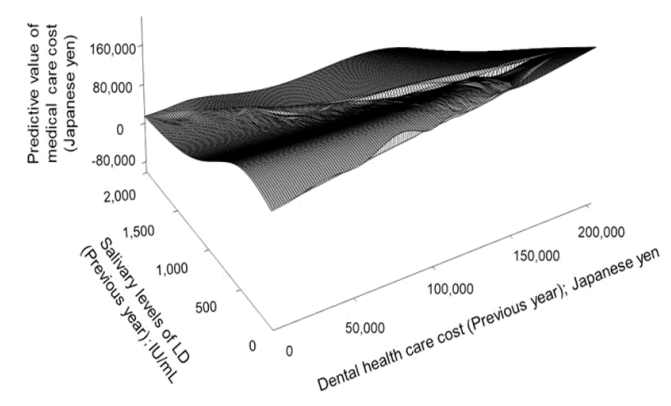

(D)

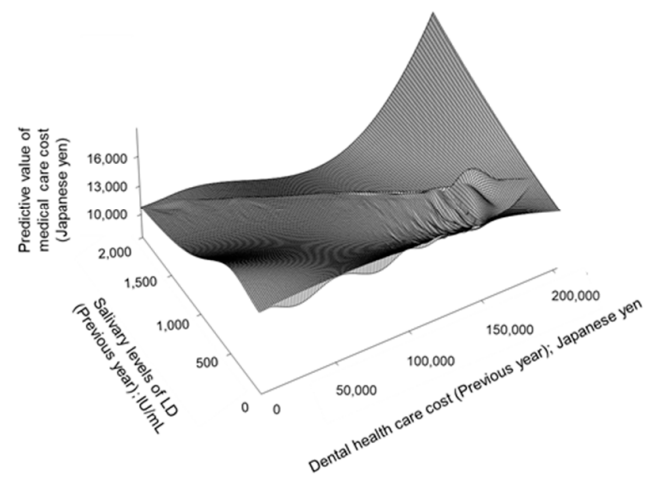

(B)

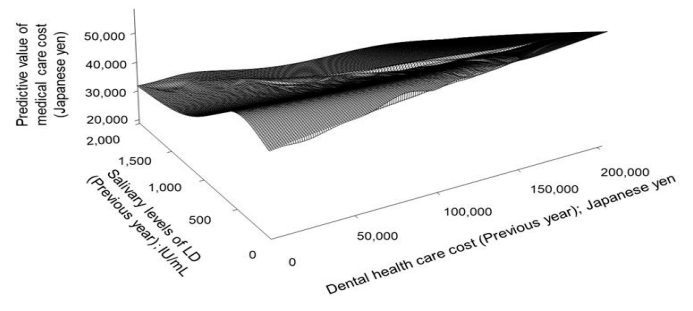

(C)

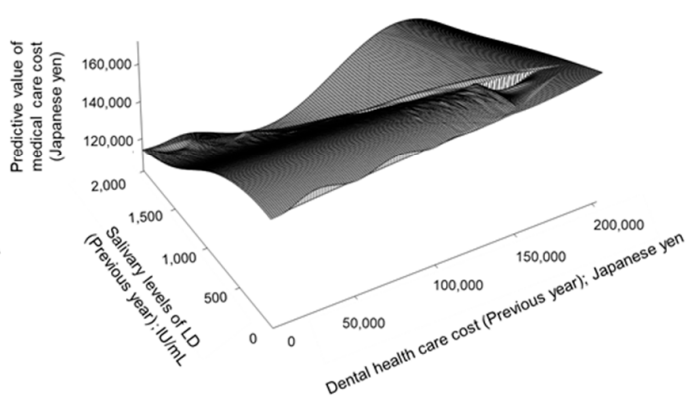

(E)

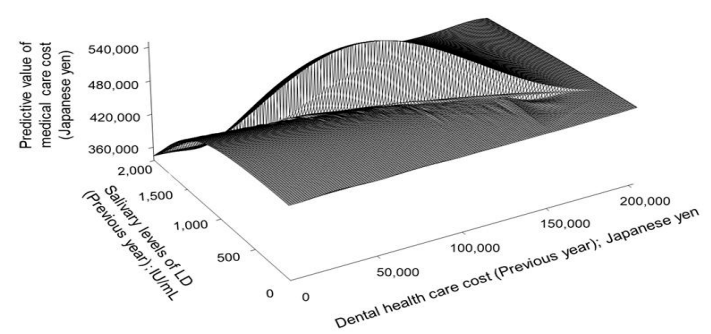

(F)

Figure 5. (A) Prediction model for medical health care costs; (B) medical health care costs from the previous year $=0$ (Japanese Yen); (C) medical health care costs from the previous year $=100,000$ (Japanese Yen); (D) medical health care costs from the previous year = 200,000 (Japanese Yen); (E) medical health care costs from the previous year $=500,000$ (Japanese Yen); $(\mathbf{F})$ medical health care costs from the previous year $=1,000,000$ (Japanese Yen). 


\section{Discussion}

The main benefits of dental treatment are improvements in oral functions and quality of life. Therefore, the outcomes of dental treatment are patients' subjective evaluation of symptoms, pain, chewing ability, comfort, and aesthetics based on the quality of life evaluation [17,18]. Therefore, dental economic analyses have been focused on the quality of life for different dental conditions. Recently, periodontal disease has been proven to be a noncommunicable disease. The prevalence or incidence of noncommunicable disease can have important outcomes on dental treatment [5,7]. However, even today, there is little information on this analysis evaluated by health care costs.

Many countries provide publicly funded medical care; however, dental care is predominantly provided by the private sector on a fee-for-service basis. For dental care, the cost is the predominant factor limiting access to care. Access to dental care is heavily influenced by the income and insurance system [19-21]. For countries under these conditions, analysis of medical health care costs and dental care costs may be biased by the economic status of the population. In Japan, the national insurance system covers almost all of the medical and dental treatments. In addition, fee-free medical and dental treatments are provided for low-income people at the same treatment levels covered by the national insurance system. Therefore, our data may contain biases derived from low income levels.

From the simulation, for all of the response surfaces from the health care costs and medical health care costs, large pits around the diagonal line from salivary levels of lactate dehydrogenase (LD) and dental health care costs can be observed. A clear mountain wave and external waviness can be observed in Figure 4A. These results indicate that the pit is not made from a single factor. There may be a balance between factors. High salivary levels of LD indicated that the oral conditions in these subjects, especially for periodontal tissues, were not good. The subjects located around the salivary levels of the LD axis are the subjects with high salivary levels of LD and have no dental health care cost expenses. These subjects may not be concerned with oral health. In addition, these subjects may not have any health care concerns. Therefore, these subjects may not comply with dental early detection and treatment and with medical treatment. These subjects may take action after symptoms become severe conditions. Therefore, health care costs may be increased. The subjects located around the dental health care costs axis are the subjects who maintain good oral conditions by professional oral care and instructed oral health care. These subjects may be well-concerned with health care and have good health conditions. Therefore, the increase in health care costs is almost flat or low with the increase in dental health care costs. Around the dental health care cost axis, shown in Figure 4A,B, predictive values of health care costs increased with the increase in dental health care costs. As health care costs include dental health care costs, the increase in health care costs was derived from the increase in dental health care costs. In contrast, health care costs decreased with increasing dental health care costs for the subjects with high salivary levels of LD. This tendency indicated that dental treatments contribute to the improvement in systemic health status.

The average health care costs for Japanese people were 333,000 yen (3027 USD) per year [1]. As shown in Figure 4A-C, predictive values of health care costs increased with increased dental health care costs and with deteriorated periodontal conditions. These subjects were relatively healthy. As a result of the simulation shown in Figure 4B-D or Figure 4E, subjects with severe periodontal disease conditions, indicated by high saliva LD levels, had decreased health care costs with increasing dental costs.

These simulations may indicate that oral hygiene treatments and promotion of oral health lead to improved general health. The results of this research may be useful for policy makers.

\section{Conclusions}

Nonlinear relationships were observed between medical health care costs, dental health care costs, and periodontal conditions. The medical health care costs from the previous year were the most important predictor for health care costs. Improvements in periodontal conditions and dental health care may play some role in reducing health care costs. 
Author Contributions: Y.N. performed the data analysis, designed the article, and wrote the manuscript. T.S. and Y.K. provided the idea for the study and collected the data. S.S. provided the idea for the study and managed the data. A.O. was a major contributor in writing the manuscript. R.O. was a major contributor in analyzing the data. E.K. provided the idea for the study and helped to draft the article. N.H. contributed to the design of the study. All authors read and approved the final manuscript.

Funding: There was no funding or financial support for this study.

Conflicts of Interest: There are no financial or nonfinancial competing interests.

Ethics Approval and Consent to Participate: The study was approved by the Ethics Committee of Tsurumi University, School of Dental Medicine (approval number: 1631) and was conducted in accordance with the Declaration of Helsinki.

Availability of Data and Material: The datasets used and/or analyzed during the current study are available from the corresponding author on reasonable request.

\section{Abbreviations}

LD: Lactate dehydrogenase

\section{References}

1. Ministry of Health, Labour and Welfare Japan. Estimates of National Medical Care Expenditure 2017. Available online: https://www.mhlw.go.jp/toukei/list/37-21.html (accessed on 15 March 2019).

2. Hirotomi, T.; Yoshihara, A.; Ogawa, H.; Miyazaki, H. Number of teeth and 5-year mortality in an elderly population. Community Dent Oral Epidemiol. 2015, 43, 226-231. [CrossRef] [PubMed]

3. Hayasaka, K.; Tomata, Y.; Aida, J.; Watanabe, T.; Kakizaki, M.; Tsuji, I. Tooth loss and mortality in elderly Japanese adults: Effect of oral care. J. Am. Geriatr. Soc. 2013, 61, 815-820. [CrossRef] [PubMed]

4. Holmlund, A.; Holm, G.; Lind, L. Number of teeth as a predictor of cardiovascular mortality in a cohort of 7674 subjects followed for 12 years. J. Periodontol. 2010, 81, 870-876. [CrossRef] [PubMed]

5. Jin, L.J.; Lamster, I.B.; Greenspan, J.S.; Pitts, N.B.; Scully, C.; Warnakulasuriya, S. Global burden of oral diseases: Emerging concepts, management and interplay with systemic health. Oral Dis. 2016, 22, 609-619. [CrossRef] [PubMed]

6. Gaffen, S.L.; Herzberg, M.C.; Taubman, M.A.; Van Dyke, T.E. Recent advances in host defense mechanisms/therapies against oral infectious diseases and consequences for systemic disease. Adv. Dent. Res. 2014, 26, 30-37. [CrossRef] [PubMed]

7. Enwonwu, C.O.; Salako, N. The periodontal disease-systemic health-infectious disease axis in developing countries. Periodontol. 2000 2012, 60, 64-77. [CrossRef] [PubMed]

8. Ministry of Health, Labour and Welfare Japan, A Basic Direction for Comprehensive Implementation of National Health Promotion. Available online: https://www.mhlw.go.jp/file/06-Seisakujouhou-10900000Kenkoukyoku/0000047330.pdf (accessed on 15 March 2018).

9. Sullan, R.M.; Li, J.K.; Crowley, P.J.; Brady, L.J.; Dufrêne, Y.F. Binding forces of Streptococcus mutans P1 adhesin. ACS Nano 2015, 9, 1448-1460. [CrossRef] [PubMed]

10. Fernandes, C.P.; Oliveira, F.A.; Silva, P.G.; Alves, A.P.; Mota, M.R.; Montenegro, R.C.; Burbano, R.M.R.; Seabra, A.D.; Lobo Filho, J.G.; Lima, D.L.F.; et al. Molecular analysis of oral bacteria in dental biofilm and atherosclerotic plaques of patients with vascular disease. Int. J. Cardiol. 2014, 174, 710-712. [CrossRef] [PubMed]

11. Kesavalu, L.; Lucas, A.R.; Verma, R.K.; Liu, L.; Dai, E.; Sampson, E.; Progulske-Fox, A. Increased atherogenesis during Streptococcus mutans infection in ApoE-null mice. J. Dent. Res. 2012, 91, 255-260. [CrossRef] [PubMed]

12. Mang-de la Rosa, M.R.; Castellanos-Cosano, L.; Romero-Perez, M.J.; Cutando, A. The bacteremia of dental origin and its implications in the appearance of bacterial endocarditis. Med. Oral Patol. Oral Cir. Bucal 2014, 19, e67-e74. [CrossRef] [PubMed]

13. Nomura, Y.; Okada, A.; Kakuta, E.; Gunji, T.; Kajiura, S.; Hanada, N. A new screening method for periodontitis: An alternative to the community periodontal index. BMC Oral Health 2016, 1, 64. [CrossRef] 
14. Nomura, Y.; Shimada, Y.; Hanada, N.; Numabe, Y.; Kamoi, K.; Sato, T.; Gomi, K.; Arai, T.; Inagaki, K.; Fukuda, M.; et al. Salivary biomarkers for predicting the progression of chronic periodontitis. Arch. Oral Biol. 2012, 57, 413-420. [CrossRef]

15. Nomura, Y.; Tamaki, Y.; Tanaka, T.; Arakawa, H.; Tsurumoto, A.; Kirimura, K.; Sato, T.; Hanada, N.; Kamoi, K. Screening of periodontitis with salivary enzyme tests. J. Oral Sci. 2006, 48, 177-183. [CrossRef] [PubMed]

16. Said, M.M.; Otomaru, T.; Sumita, Y.; Leung, K.C.M.; Khan, Z.; Taniguchi, H. Systematic review of literature: Functional outcomes of implant-prosthetic treatment in patients with surgical resection for oral cavity tumors. J. Investig. Clin. Dent. 2017, 8, e12207. [CrossRef]

17. Kakuta, E.; Nomura, Y.; Naono, Y.; Koresawa, K.; Shimizu, K.; Hanada, N. Correlation between health-care costs and salivary tests. Int. Dent. J. 2013, 63, 249-253. [CrossRef]

18. Karlsson, G.; Teiwik, A.; Lundström, A.; Ravald, N. Costs of periodontal and prosthodontic treatment and evaluation of oral health in patients after treatment of advanced periodontal disease. Community Dent. Oral Epidemiol. 1995, 23, 159-164. [CrossRef]

19. Millar, W.J.; Locker, D. Dental insurance and use of dental services. Health Rep. 1999, 11, 55-67.

20. Ramraj, C.; Quiñonez, C.R. Self-reported cost-prohibitive dental care needs among Canadians. Int. J. Dent. Hyg. 2013, 11, 115-120. [CrossRef] [PubMed]

21. Thompson, B.; Cooney, P.; Lawrence, H.; Ravaghi, V.; Quiñonez, C. The potential oral health impact of cost barriers to dental care: Findings from a Canadian population-based study. BMC Oral Health 2014, 14, 78. [CrossRef] [PubMed]

(C) 2020 by the authors. Licensee MDPI, Basel, Switzerland. This article is an open access article distributed under the terms and conditions of the Creative Commons Attribution (CC BY) license (http://creativecommons.org/licenses/by/4.0/). 